\title{
CHARACTERISTIC MODEL OF A SHOCK ABSORBER IN AN UNMANNED GROUND VEHICLE
}

\author{
Ing. Ján Danko, PhD. ${ }^{1}$, Ing. Tomáš Milesich ${ }^{1}$, Ing. Martin Bugár ${ }^{2}$, Ing. Juraj Madarás ${ }^{2}$
}

${ }^{1}$ Slovak university of technology in Bratislava, Faculty of mechanical engineering, Nám. Slobody 17,81231 Bratislava, jan.danko@stuba.sk, tomas.milesich@stuba.sk

${ }^{2}$ Slovak university of technology in Bratislava, Faculty of electrical engineering and information technology, Ilkovičova 3,81219 Bratislava, martin.bugar@stuba.sk, juraj.madaras@stuba.sk

Keywords: Suspension system. Shock absorber. Hysteresis. Bouc-Wen model

\begin{abstract}
The paper deals with mathematical models for the shock absorber of an unmanned ground vehicle. The possibility of mathematically modeling the shock absorber is discussed. Specific types of mathematical models are described and the experimental measurement of a shock absorber is made. For modeling the characteristics of the shock absorber the modified Bouc-Wen model (Spencer model) is selected. From the mathematical model, a simulation model in Matlab/Simulink is created. The identification of the Spencer model parameters is performed and force-velocity and force-displacement characteristics of the shock absorber of an unmanned ground vehicle is made. In the conclusions, the simulated characteristics are verified and evaluated by the measured characteristics.
\end{abstract}

\section{INTRODUCTION}

Unmanned ground vehicles (UGV) can be used in many conditions. The suspension of UGV's can undergo highly demanding situations, especially in terms of driving safety and ride comfort. Driving safety and ride comfort are important for the safe operation of the electronic control systems. To achieve proper suspension characteristics, it is important in the design of the vehicle to ensure the appropriate parameters and characteristics of the suspension components to be used [1].

In order to reduce costs, virtual models are used, which must ensure a high compliance with real world conditions. In terms of suspension, it is necessary that the virtual model of the UGV must contain the most comprehensive model of the suspension components, which provide relevant results and facilitate the testing and examination of the proposed vehicle [1,2]. Therefore, models of suspension components such as the spring, shock absorber, bushings and mountings are based on mathematical equations that describe the most complex behavior of each component [3]. This paper deals with the modeling of the shock absorber characteristics.

Models of a shock absorber can be, in terms of the characteristics of the model, divided into parametric and nonparametric parts. Nonparametric models are based on the characteristics of the device. This paper deals with parametric models. Parametric models simulate the response of power quantitatively. Models are adapted to the result of the experimental measurements by modifying their parameters. Models are represented by a mathematical function. Most parametric models are described by assembling mechanical components such as spring and viscous shock absorber together. 


\section{PARAMETRIC MODELS}

One of the parametric models for the elastic components is the Kelvin-Voight component in figure 1. It consists of a parallel sequence with a spring and shock absorber. The spring has a linear stiffness $k$ and shock absorber with dumping $c$. In point $\mathrm{C}$ the kinematic exciting $x(t)$ is generated and in point $\mathrm{B}$ is the force response $\mathrm{F}_{\mathrm{B}}$. figure 2 is shown the characteristic of component.

$$
F_{z}=k x(k)+\operatorname{cx}(k)
$$

Due to the high nonlinearity between the measured characteristics of the specific components, the Kelvin-Voight model is inadequate.

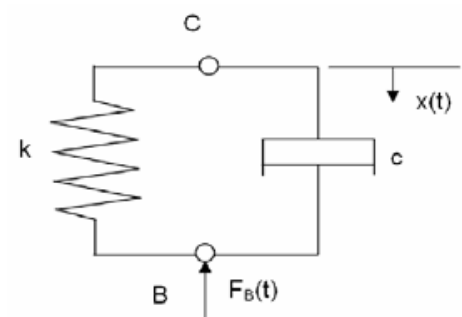

Figure 1: Kelvin-Voight component

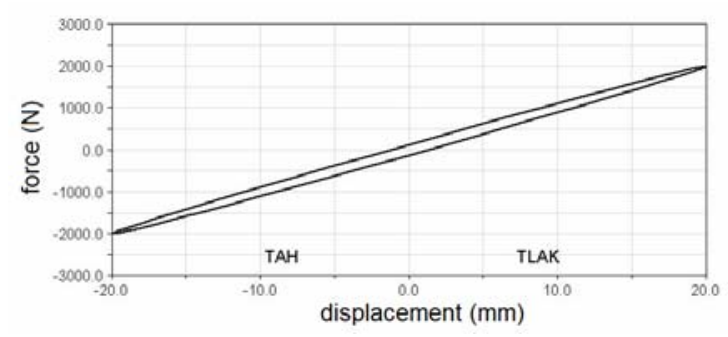

Figure 2: Force-displacement graph

Therefore the Bouc-Wen model is used, which approximates the measured characteristics better than simple force components. The model involves an inherent simplicity. Different types of shock absorber characteristics can be represented using just the Bouc-Wen model. The shape of the Bouc-Wen hysteretic loop depends on four parameters $\beta, \mathrm{n}, \gamma$ and $\mathrm{A}$. The model is based on a nonlinear ordinary differential equation which contains a memory variable $z$, figure $3,[6]$.

The Bouc-Wen model is defined by the hysteresis variable:

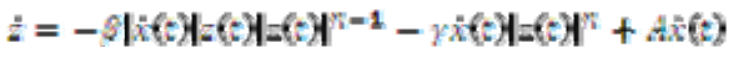

where $z$ is a variable of the hysteresis and the dot denotes the time derivative, $x(t)$ is the imposed displacement of the device (or the material deformation - model of a bushing or same elastic component) [4].

The power produced by the hysteresis component represented by the Bouc-Wen model, is expressed as follows:

$$
F_{z w}(t)=a \frac{F_{y}}{x_{y}} x(t)+(1-a) F_{y} z(t)
$$

The quantities $\beta, \gamma, n, A$ and $a$ are loop parameters and parameters $x_{y}, F_{y}$ describe the hysteresis component in the Bouc-Wen model.

The resulting force response of the model is: 


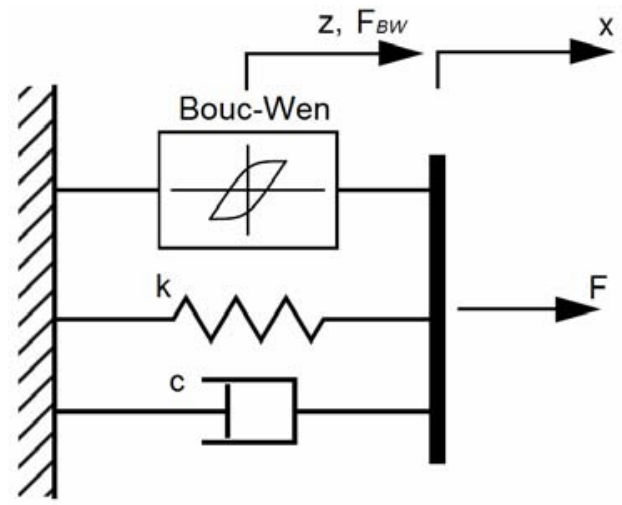

Figure 3: Bouc-Wenov model of the elastic component

A specific characteristic feature obtained from the results of the model was the ability to model using the modified Bouc-Wen's model, according to Spencer [5], which is shown in figure 4.

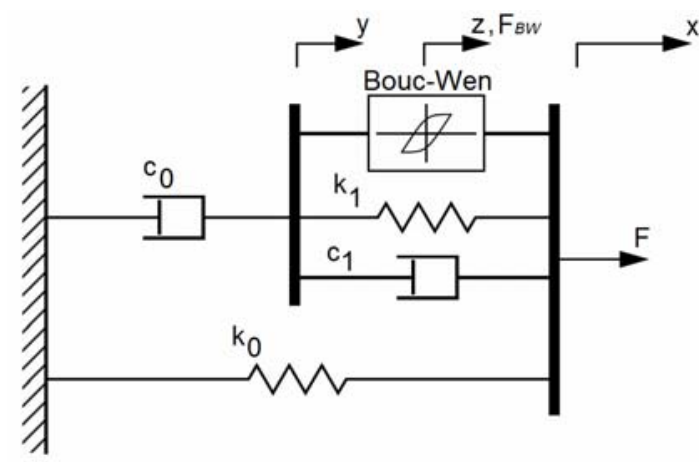

Figure 4: The modified Bouc-Wen model by Spencer

The equations defining the model are:

$$
\begin{aligned}
& \varsigma_{0} y=\alpha_{z}+\kappa_{2}(x-y)+\varsigma_{1}(x-y)
\end{aligned}
$$

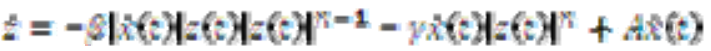

$$
\begin{aligned}
& y=\frac{1}{\left(c_{1}+c_{0}\right)}\left(\kappa z+s_{1} k+k_{1}(x-y)\right) \\
& F=a z+\varepsilon_{2}(x-y)+k_{2}(x-y)+k_{0}\left(x-x_{0}\right)
\end{aligned}
$$


Where $\approx$ is the loop parameter from the Spencer model and $\mathrm{x}_{0}$ is the initial displacement of the spring $\mathrm{k}_{0}$. The resulting force response is:

$$
F=\varepsilon_{0} x+\kappa_{0}\left(x-x_{0}\right)
$$

\section{EXPERIMENTAL MEASUREMENT}

Experimental measurements have been performed on the EDYZ test bed. The measured shock absorber was kinematically excited by an excitation device. A sinusoidal signal was used for excitation.

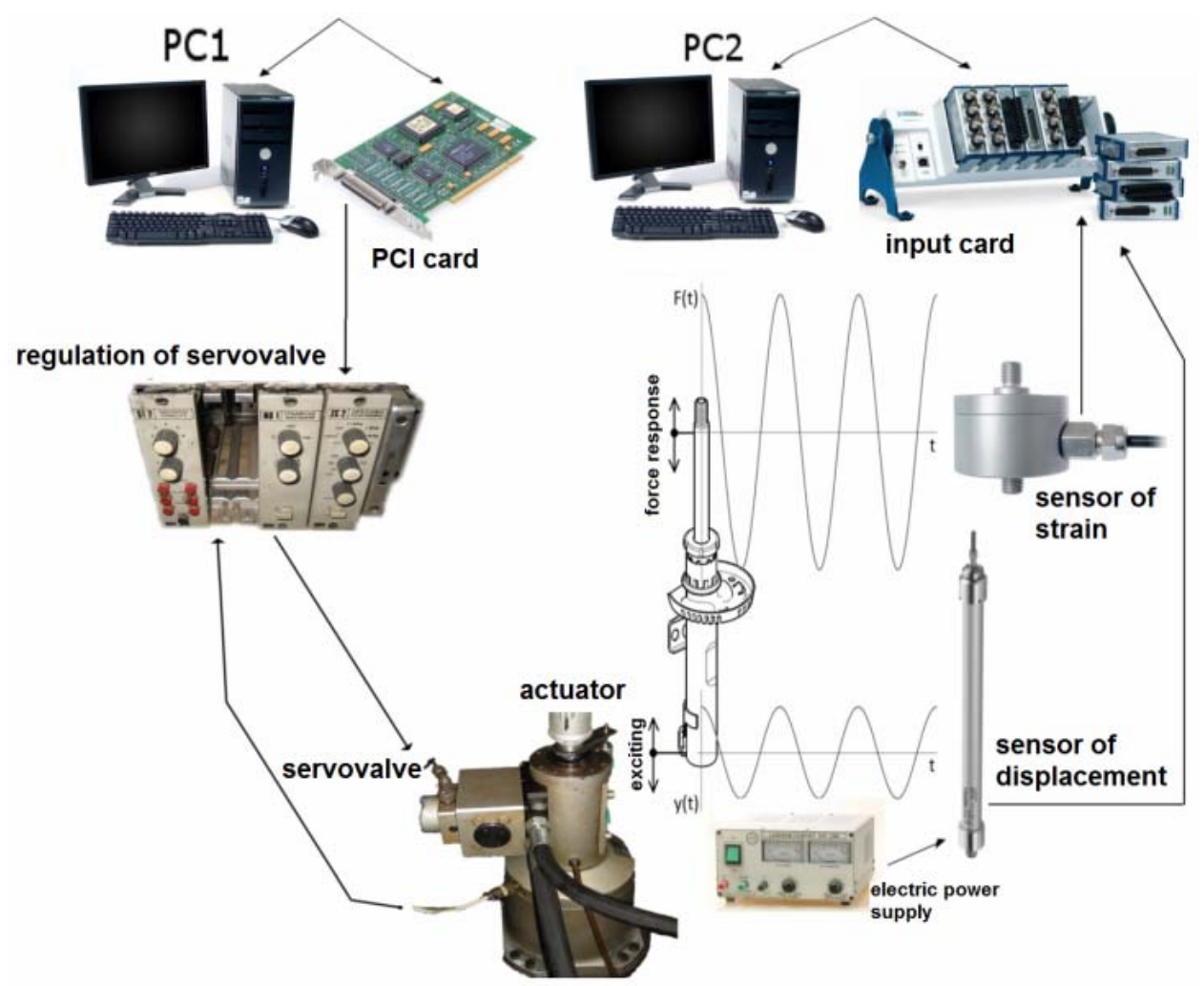

Figure 5: Schematic diagram of measurement and excitation components

The measurements are carried out according to the scheme in figure 5, which schematically shown the involvement of measuring input cards and output PCI card. The output PCI card generates a signal and sends it to the servovalve. Force response is acquired by a tensometer. The signal from the potentiometer and tensometer is recorded through the input card in PC2, which was processed in LabVIEW Signal Express.

\section{MODELLING OF SHOCK ABSORBER IN MATLAB/SIMULINK}


Modeling of the shock absorber was done in MATLAB / Simulink. In Simulink, the complete modified Buoc-Wen model was created.

\subsection{Identification of model parameters}

Model parameters are determined through the optimization toolbox for parameter identification Control and Estimation Tools Manager in Simulink. First, the course of power response is loaded into the toolbox, which was obtained from experimental measurements in the shape $(\mathrm{Nx} 1)$ for values of power and also for time values. Due to the resulting process, the parameters of differential equation of the model were argued.

\subsection{Modeled characteristics of the shock absorber}

After identifying the model parameters the characteristics of the shock absorber were made. Characteristics were modeled for various amplitudes, displacements and different frequency of the excitation signal. In figure 6 characteristics of modeled and measured data are presented.

- Modeled and measured characteristics of the shock absorber:
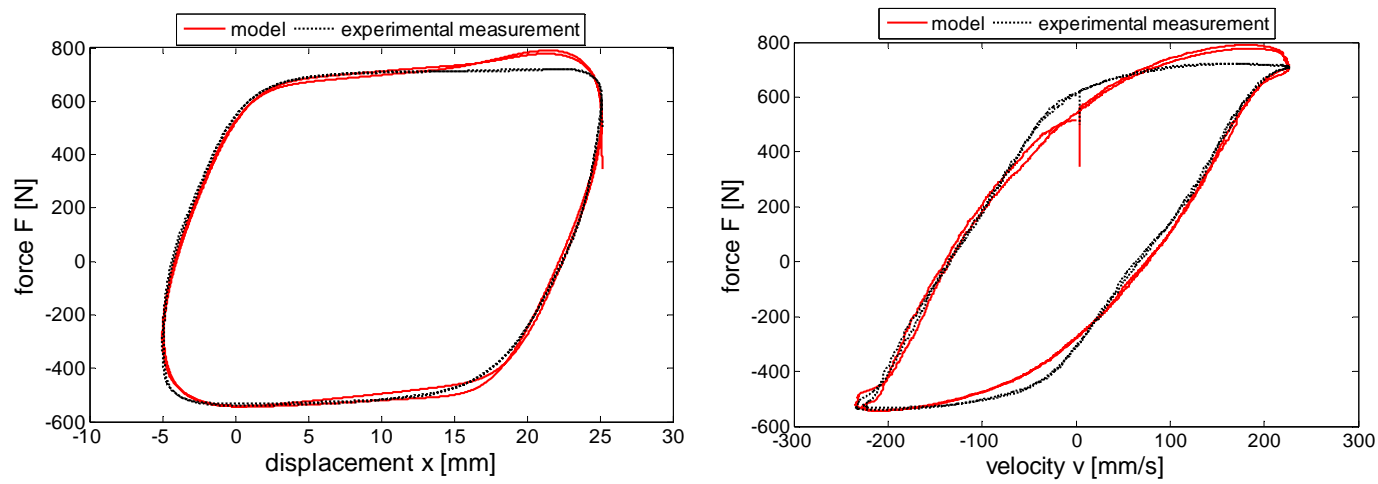

Figure 6: Force-displacement and force-velocity characteristics of the shock absorber at a frequency of $2.5 \mathrm{~Hz}$ and amplitude of $15 \mathrm{~mm}$

\subsection{Evaluation of the modeled and measured characteristics of the shock absorber}

The error of model $\varepsilon$ is determined by comparing the power response of the experimental measurement and force response of the model according to equation (10),

$$
a=\sum_{t=1}^{n} \frac{\left|F_{r_{i}}-F_{m_{i}}\right|}{n}
$$


where $n$ is the number of lines in matrix, $\mathbf{F}_{\hat{A}_{1}}$ is the $\mathrm{i}$-th value of the experimental measured force and $\mathrm{F}_{\mathrm{m}_{1}}$ is the $\mathrm{i}$-th value of measured force from model. Calculating the percentage deviation of

the conformity of the model $\sigma$ experiment is performed according to our proper equation (11).

$$
z=\frac{z}{\frac{\sum_{i-1}\left|F_{e}\right|}{n}}+100
$$

Table 1: Errors of modeled and measured forces

\begin{tabular}{|c|c|c|c|c|c|}
\hline $\mathrm{f}(\mathrm{Hz})$ & 0,25 & 0,5 & 1 & 2,5 & 5 \\
\hline$\varepsilon(\mathrm{N})$ & 4,5 & 6,1 & 20 & 27 & 42,2 \\
\hline$\sigma(\%)$ & 4,4 & 3,7 & 6,6 & 5,5 & 6,9 \\
\hline
\end{tabular}

The graphs show that the modified Bouc-Wen model can be used to modeling the characteristics of the suspension components of any kind. Problem remains the identification of model parameters. In table 1 are presented results of errors of modeled and measured forces. From results of model evaluation is shown that model of hysteresis is approximated characteristics obtained from measure nearly ideally. Deviation is caused by the identification of model parameters. Identification of parameters is specific and required to create their own method of optimization.

\section{CONCLUSION}

Modeling of shock absorber characteristics of UGV is difficult in terms of building a precise virtual model of suspension respectively of full body vehicle model of UGV. Modified Bouc-Wen model used in simulation shown good results via wide range of input frequencies and amplitudes. It is difficult identify parameters of proposed model. Control and estimation toolbox used by identification of model parameters here show good results by certain frequencies and amplitudes. For better compliance between modeled and measured data will be needs designed own method of parameter identification or use some better identification methods. In next the virtual full body model of UGV will be designed with these model characteristics of suspension components for investigation of vertical dynamics and for estimation of the optimal suspension characteristics.

\section{ACKNOWLEDGMENT}

This work was supported by the Slovak university of technology under The program for support of young research workers 


\section{REFERENCES}

[1] Dixon J. C.: The Shock Absorber Handbook. John Wiley \& Sons Ltd. 2007 s. 455. ISBN 978$0-470-51020-9$

[2] Heißing B., Ersoy M.: Chassis Handbook. Springer Fachmedien Wiesbaden GmbH 2011. ISBN 978-3-8348-0994-0

[3] Bigoš P., Puškár, M., Kopas, M.,: Design of Mechatronic System Specified for Feedback Required in Development of Combustion Engines, Procedia Engineering, Volume 48, 2012, Pages 30-34, ISSN 1877-7058

[4] Worden K., Manson G.: On the Identification of Hysteretic Systems. Society for Experimental Mechanics Inc, 2010. ISBN 978-1-4419-9718-0

[5] B. F. Spencer Jr., S. J. Dyke, M. K. Sain, J. D. Carlson: Phenomenological Model of a Magnetorheological Damper. Journal of Engineering Mechanics. 1997. 230-238 ISSN: 07339399

[6] Guglielmino E., Sireteanu T., Stammers Ch. W., Ghita G., Giuclea M.: Semi-active Suspension Control, Springer-Verlag London Limited 2008. s 302. ISBN 978-1-84800-230-2 